Research Paper

\title{
When to apply immune checkpoint inhibitor in patients with refractory advanced gastric cancer
}

Hongsik Kim, Hana Kim, Minsang Lee, Minsuk Kwon, Jung Yong Hong, Jeeyun Lee, Ho Yeong Lim, Won Ki Kang, Seung Tae Kim ${ }^{\bowtie}$

Division of Hematology-Oncology, Department of Medicine, Samsung Medical Center, Sungkyunkwan University School of Medicine, Seoul, Republic of Korea

$\triangle$ Corresponding author: Seung Tae Kim, Division of Hematology-Oncology, Department of Medicine, Samsung Medical Center, Sungkyunkwan University School of Medicine, 81 Irwon-ro, Gangnam-gu, Seoul 06351, Republic of Korea. Tel: 82-2-3410-1779; Fax: 82-2-3410-1754; E-mail: shty1@skku.edu

(C) The author(s). This is an open access article distributed under the terms of the Creative Commons Attribution License (https://creativecommons.org/licenses/by/4.0/). See http:/ /ivyspring.com/terms for full terms and conditions.

Received: 2021.05.17; Accepted: 2021.07.18; Published: 2021.07.25

\begin{abstract}
Background: Immune checkpoint inhibitors (ICls) show clinical benefit in patients with refractory advanced gastric cancer (GC). The ICls in routine clinical practice have been used in various treatment lines. Therefore, we investigated the timing for application of $\mathrm{ICl}$ in patients with refractory advanced $\mathrm{GC}$.

Methods: We analyzed 187 patients with refractory advanced or recurrent GC who received ICIS as a 3rd- or 4th-line treatment between September 2015 and October 2020. Clinical outcomes of overall survival (OS), progression-free survival (PFS), objective response rate (ORR), and disease control rate (DCR) were evaluated.

Results: Among 187 patients, 105 received ICls as a 3rd-line treatment and 82 as a 4th line. The ORR for ICls was $10.5 \%(11 / 105)$ in $3 \mathrm{rd}$ line and $8.5 \%(7 / 82)$ in 4 th line. The DCR for ICls was $36.2 \%(38 / 105)$ in 3 rd-line treatment and $31.7 \%(26 / 82)$ in 4th line. There was no significant difference for ORR $(P=0.819)$ or DCR $(P=0.870)$. The median PFS and OS to ICls was 1.4 months $(95 \% \mathrm{Cl}, 1.1$ to 1.8 months) and 4.4 months (95\% Cl, 1.6 to 7.2 months) in 3 rd line and 1.8 months $(95 \% \mathrm{Cl}, 1.4$ to 2.3 months) and 2.8 months ( $95 \% \mathrm{Cl}, 2.2$ to 3.4 months) in 4th line. The median PFS and OS to ICls was not different between 3 rd line and 4th line $(P=0.495$ and $P=0.208$, respectively). There were also no significant difference for PFS and OS between PD-L1-positive tumors (CPS $\geq 1)$ and PD-L1-negative tumors $(P=0.910$ and $P=0.931$, respectively).

Conclusions: ICls showed similar clinical benefits in the 3rd-line and 4th-line settings. ICls might be a reasonable approach for patients with refractory GC in the setting of 3rd-line or 4th-line treatment options.
\end{abstract}

Key words: Gastric cancer, Immune checkpoint inhibitor, Nivolumab, Pembrolizumab, Biomarker

\section{Introduction}

Gastric cancer (GC) is the sixth most common cancer and the second leading cause of cancer death worldwide [1]. In Korea, GC is the most incident cancer and the fourth leading cause of cancer death [2]. In National Comprehensive Cancer Network (NCCN) and European Society for Medical Oncology (ESMO) guidelines, palliative chemotherapy is a standard care in patients with metastatic GC. The standard first-line chemotherapy is the combination of platinum and pyrimidine analogues, and common second lines are a combination of paclitaxel and ramucirumab, paclitaxel, docetaxel or irinotecan $[3,4]$. Despite these treatments, most patients with advanced GC experience disease progression, and prognosis is very poor.

Recently, immune checkpoint inhibitors (ICIs) showed clinical benefit in patients with refractory advanced GC [5]. The ATTRACTION-2 (ONO4538-12) study showed clinical benefit of nivolumab, a human IgG4 monoclonal antibody inhibitor of programmed death-1 (PD-1), in GC patients who had failed two or more treatments. The objective response 
rate (ORR) of nivolumab was $11.2 \%$, and median overall survival (OS) showed improvement compared to placebo (5.3 versus 4.1 months), regardless of positivity for programmed death-ligand 1 (PD-L1) [6]. Also, the KEYNOTE-059 study reported the clinical benefit of pembrolizumab, another human IgG4 monoclonal antibody inhibitor of PD-1, in refractory GC patients as salvage therapy. The ORR of pembrolizumab was $11.6 \%$ in all enrolled patients and $15.5 \%$ in patients with PD-L1-positive tumors [7].

However, the optimal timing of the application of ICIs has not been determined, and numerous clinical trials are on-going. In clinical practice, the ICIs in routine clinical practice have been used in various treatment lines.

Herein, we investigated the timing of application of ICI in patients with refractory advanced GC.

\section{Patients and methods}

\section{Patients}

From September 2015 to October 2020 at Samsung Seoul Medical Center, 187 refractory GC patients treated with ICIs after progression on 2nd- or 3rd-line therapy were analyzed in this study. The following clinicopathologic characteristics were collected for all 187 patients: age, sex, number of metastatic sites, microsatellite instability (MSI), Epstein-Barr virus (EBV) in situ, positivity for PD-L1, and information on chemotherapy. The study protocol was approved (\#2021-01-053) by the Institutional Review Board of Samsung Medical Center (Seoul, Korea), and was conducted in accordance with the ethical principles of the Declaration of Helsinki and the Korea Good Clinical Practice guidelines.

\section{Chemotherapy}

Patients included in this study received nivolumab or pembrolizumab as ICI according to the physician. Nivolumab was administered at a dose of $3 \mathrm{mg} / \mathrm{kg}$ intravenously every 2 weeks or pembrolizumab at a dose of $200 \mathrm{mg}$ intravenously every 3 weeks.

\section{Statistics}

Descriptive statistics were used to summarize patient and tumor characteristics. Categorical variables were analyzed by chi-square test. Survival analyses were performed using the Kaplan-Meier method, and differences were analyzed by log-rank test. Hazard ratios and corresponding 95\% confidence intervals were calculated using the Cox proportional hazards model. Progression-free survival (PFS) was defined as the time from the start of ICI until the date of disease progression or death from any cause.
Univariate analysis was performed using Cox proportional hazards models for PFS and OS. Significant prognostic variables in univariate analysis for survival were included in the multivariate analysis. All $P$-values were two-sided, and statistical significance was set at $P<0.05$. Statistical analysis was performed using IBM SPSS statistics version 25 .

\section{Results}

\section{Patient characteristics}

Between September 2015 and October 2020, we analyzed 187 patients with refractory advanced or recurrent GC with immune checkpoint inhibitor (ICI), of whom 105 received ICI as a 3rd line and 82 received ICI as a 4th line treatment. Patient characteristics are presented in Table 1. Of patients with ICI as 3rd line, the median age was 57 years (range of 27 to 82 years), and $89(84.8 \%)$ patients had two or more distant metastatic sites. Of patients with ICI as 4 th line, the median age was 57 years (range of 29 to 78 years), and $80(97.6 \%)$ patients had two or more distant metastatic sites. Status of MSI-H and EBV positivity were not different in patients with ICIs of 3rd or 4th line. However, positivity of PD-L1 expression was significantly higher in patients with ICI as 3rd line than in those with ICI as 4th line.

Table 1. Patient characteristics

\begin{tabular}{|c|c|c|c|}
\hline Patient characteristics & $\begin{array}{l}\text { 3rd line }(n= \\
105)\end{array}$ & $\begin{array}{l}\text { 4th line }(n= \\
82)\end{array}$ & $P$-value \\
\hline Median age (years) & $57(27-82)$ & $57(29-78)$ & 0.349 \\
\hline Patients aged $<65$ years & $76(72.4 \%)$ & $64(78.0 \%)$ & 0.375 \\
\hline Sex & & & 0.209 \\
\hline Male & $57(54.3 \%)$ & $52(63.4 \%)$ & \\
\hline Female & $48(45.7 \%)$ & $30(36.6 \%)$ & \\
\hline Immune checkpoint inhibitor & & & 0.156 \\
\hline Nivolumab & $72(68.6 \%)$ & $48(58.5 \%)$ & \\
\hline Pembrolizumab & $33(31.4 \%)$ & $34(41.5 \%)$ & \\
\hline Organs with metastases & & & 0.003 \\
\hline$<2$ & $16(15.2 \%)$ & $2(2.4 \%)$ & \\
\hline$\geq 2$ & $89(84.8 \%)$ & $80(97.6 \%)$ & \\
\hline \multicolumn{4}{|l|}{ Previous therapies } \\
\hline Any & $105(100 \%)$ & $82(100 \%)$ & \\
\hline Pyrimidine analogues & $105(100 \%)$ & $82(100 \%)$ & \\
\hline Platinum & $102(97.1 \%)$ & $80(97.6 \%)$ & \\
\hline Taxane & $90(85.7 \%)$ & $81(98.8 \%)$ & \\
\hline Irinotecan & $14(13.3 \%)$ & $68(82.9 \%)$ & \\
\hline Ramucirumab & $76(72.4 \%)$ & $60(73.2 \%)$ & \\
\hline Microsatellite instability & & & 0.381 \\
\hline MSS & $84(80.0 \%)$ & $64(78.0 \%)$ & \\
\hline MSI-H & $2(1.9 \%)$ & $0(0 \%)$ & \\
\hline Not evaluable & $19(18.1 \%)$ & $18(22.0 \%)$ & \\
\hline EBV in situ & & & 0.621 \\
\hline No & $69(65.7 \%)$ & $49(59.8 \%)$ & \\
\hline Yes & $4(3.8 \%)$ & $5(6.1 \%)$ & \\
\hline Not evaluable & $32(30.5 \%)$ & $28(34.1 \%)$ & \\
\hline PD-L1 22C3 IHC CPS & & & 0.062 \\
\hline PD-L1<1 & $21(20.0 \%)$ & $16(19.5 \%)$ & \\
\hline $1 \leq \mathrm{PD}-\mathrm{L} 1<10$ & $24(22.9 \%)$ & $5(6.1 \%)$ & \\
\hline $10 \leq \mathrm{PD}-\mathrm{L} 1$ & $8(7.6 \%)$ & $6(7.3 \%)$ & \\
\hline Not evaluable & $52(49.5 \%)$ & $55(67.1 \%)$ & \\
\hline
\end{tabular}

MSS Microsatellite stable, MSI-H Microsatellite instability-high, EBV Epstein-Barr virus, PD-L1 Programmed death-ligand 1, IHC Immunohistochemistry, CPS

Combined positive score. 

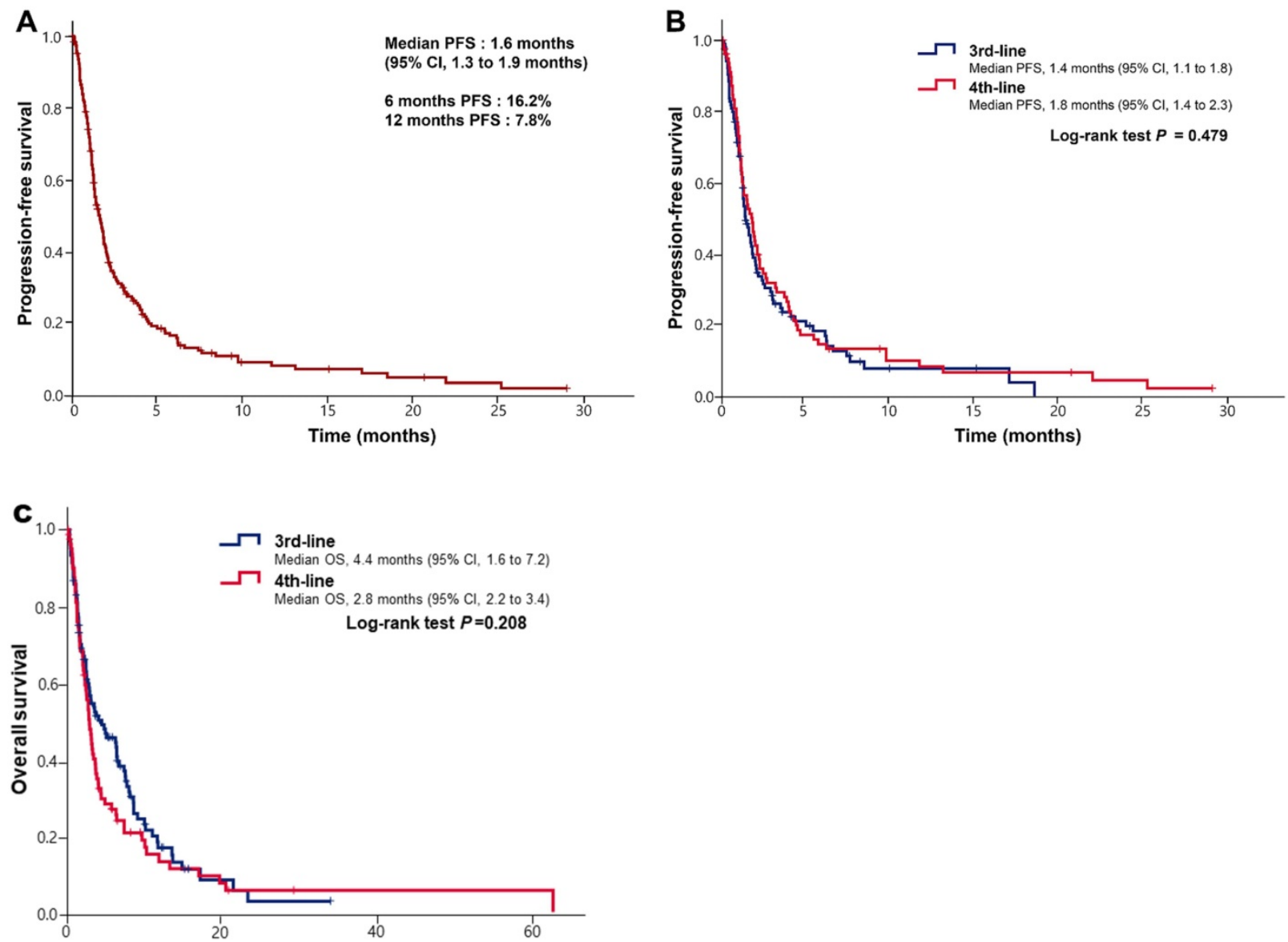

Figure 1. (A) Kaplan-Meier curves of progression-free survival (PFS) after immune checkpoint inhibitors. (B) Kaplan-Meier curves of progression-free survival (PFS) after immune checkpoint inhibitors according to lines of treatment. (C) Kaplan-Meier curves of overall survival (OS) after immune checkpoint inhibitors according to lines of treatment.

\section{Treatment outcomes with ICls between 3rd- and 4th-line treatment}

The median duration of follow-up was 3.0 months (IQR 1.4 to 7.5 months) in patients with ICI as 3rd-line treatment and 2.7 months (IQR 1.2 to 5.6 months) in those with 4 th line. The overall response rate (ORR) for ICIs was 10.5\% (11/105) in 3rd line and $8.5 \%(7 / 82)$ in 4 th line $(P=0.819)$. The DCR for ICIs was $36.2 \%(38 / 105)$ in 3rd line and $31.7 \%(26 / 82)$ in 4 th line $(P=0.870)$. Between $3 \mathrm{rd}$ and 4 th lines, there was no difference in efficacy of ICIs (Table 2).

Table 2. Objective response rate and survival data

\begin{tabular}{llll}
\hline & $\mathbf{3}^{\text {rd }}$ line $(\mathbf{n}=\mathbf{1 0 5})$ & $\mathbf{4}^{\text {th }}$ line $(\mathbf{n}=\mathbf{8 2})$ & $\boldsymbol{P}$-value \\
\hline Complete response & 0 & 0 & \\
Partial response & $11(10.5 \%)$ & $7(8.5 \%)$ & \\
Stable disease & $27(25.7 \%)$ & $19(23.2 \%)$ & \\
Progressive disease & $47(44.8 \%)$ & $34(41.5 \%)$ & \\
Not evaluable & $20(19.0 \%)$ & $22(26.8 \%)$ & 0.819 \\
Objective response rate & $11(10.5 \%)$ & $7(8.5 \%)$ & 0.870 \\
Disease control rate & $38(36.2 \%)$ & $26(31.7)$ & \\
\hline
\end{tabular}

The median PFS after ICI was 1.6 months (95\% CI, 1.3 to 1.9 months) (Figure 1A). The median PFS was 1.4 months (95\% CI, 1.1 to 1.8 months) in 3rd-line treatment and 1.8 months (95\% CI, 1.4 to 2.3 months) in 4 th-line treatment, with no statistical difference $(P=$ 0.495) (Figure 1B). The median PFS in patients with PD-L1-positive tumors (CPS $\geq 1$ ) was 2.0 months (95\% CI, 1.1 to 3.0 months), while that in negative tumors was 1.9 months (95\% CI, 1.3 to 2.5 months) $(P=0.910)$. The median OS was 4.4 months (95\% CI, 1.6 to 7.2 months) in 3rd-line treatment and 2.8 months (95\% CI, 2.2 to 3.4 months) in 4th-line treatment, with no statistical difference $(P=0.208$, Figure $1 C)$. We found that 18 of 187 patients $(9.6 \%)$ achieved the long term survival of more than 1 year. Furthermore, 7 of 187 patients $(3.7 \%)$ had received ICIs for more than 1 year, and 3 continued to do so to present. Of 18 patients with long-term survival, 2 of 12 patients $(16.7 \%)$ were EBV positivity, 4 of 9 patients $(44.4 \%)$ were PD-L1 expression and 0 of $7(0 \%)$ patients were MSI-H.

\section{Analysis of prognostic factors for PFS and OS after the starting ICls}

We conducted the univariate analysis for PFS and OS after starting ICIs to identify significant prognostic factors (Table 3, Table 4). In univariate analysis, age $(\geq 65$ vs. $<65)$ was only significant 
prognostic factor to PFS $(P=0.003)$. Age $(\geq 65$ vs. $<65)$ and No. of metastatic sites ( $<2$ vs. $\leq 2)$ were significantly associated with $\mathrm{OS}(P=0.018$ and $P=0.012$, respectively). The timing of starting ICIs as 3rd or 4th line was not associated with PFS and OS, in univariate analysis. Multivariate analysis showed that age $(P=$ $0.030)$ and No. of metastatic sites $(P=0.019)$ were significant independent prognostic factors for OS.

Table 3. Univariate analysis of progression-free survival after ICls

\begin{tabular}{|c|c|c|c|c|}
\hline \multirow[t]{2}{*}{ Variables } & \multirow[t]{2}{*}{ No. } & \multirow{2}{*}{$\begin{array}{l}\text { Median PFS } \\
\text { (months, 95\% CI) }\end{array}$} & \multicolumn{2}{|c|}{ Univariate Analysis } \\
\hline & & & HR $(95 \%$ CI) & $P$-value \\
\hline Median age, years & & & & 0.003 \\
\hline$<65$ & 140 & $1.4(1.1-1.7)$ & 1 & \\
\hline$\geq 65$ & 47 & $2.6(0.0-5.5)$ & $0.57(0.39-0.83)$ & \\
\hline Sex & & & & 0.209 \\
\hline Male & 109 & $1.5(1.2-1.9)$ & 1 & \\
\hline Female & 78 & $1.8(1.0-2.7)$ & $0.82(0.60-1.12)$ & \\
\hline Immune checkpoint inhibitors & & & & 0.502 \\
\hline $3^{\text {rd }}$ line & 105 & $1.4(1.1-1.8)$ & 1 & \\
\hline $4^{\text {th }}$ line & 82 & $1.8(1.4-2.3)$ & $0.90(0.66-1.23)$ & \\
\hline No. with metastatic sites & & & & 0.086 \\
\hline$<2$ & 18 & $3.1(0.7-5.4)$ & 1 & \\
\hline$\geq 2$ & 169 & $1.5(1.2-1.8)$ & $1.60(0.94-2.73)$ & \\
\hline Microsatellite instability* & & & & 0.327 \\
\hline MSS & 148 & $3.7(2.6-4.7)$ & 1 & \\
\hline MSI-H & 2 & $1.2(0.6-1.7)$ & $2.02(0.50-8.23)$ & \\
\hline Not evaluable & 37 & & & \\
\hline EBV in situ & & & & 0.509 \\
\hline No & 118 & $1.7(1.3-2.0)$ & 1 & \\
\hline Yes & 9 & $3.2(0.0-6.4)$ & $0.77(0.36-1.67)$ & \\
\hline Not evaluable & 60 & & & \\
\hline
\end{tabular}

\begin{tabular}{|c|c|c|c|c|}
\hline \multirow[t]{2}{*}{ Variables } & \multirow[t]{2}{*}{ No. } & \multirow{2}{*}{$\begin{array}{l}\text { Median PFS } \\
\text { (months, 95\% CI) }\end{array}$} & \multicolumn{2}{|c|}{ Univariate Analysis } \\
\hline & & & HR (95\% CI) & $P$-value \\
\hline PD-L1 22C3 IHC CPS $\geq 1$ & & & & 0.921 \\
\hline No & 37 & $1.9(1.3-2.5)$ & 1 & \\
\hline Yes & 43 & $2.0(1.1-3.0)$ & $0.97(0.59-1.60)$ & \\
\hline Not evaluable & 107 & & & \\
\hline PD-L1 22C3 IHC CPS $\geq 10$ & & & & 0.957 \\
\hline No & 66 & $1.9(1.4-2.4)$ & 1 & \\
\hline Yes & 14 & $1.3(0.1-2.4)$ & $1.02(0.54-1.92)$ & \\
\hline Not evaluable & 107 & & & \\
\hline $\begin{array}{l}\text { HR Hazard ratio, } M S S \text { Micr } \\
E B V \text { Epstein-Barr virus, } P D \\
\text { Immunohistochemistry, } C P\end{array}$ & $\begin{array}{l}\text { llite s } \\
\text { rogra } \\
\text { nbine }\end{array}$ & $\begin{array}{l}\text { stable, } M S I-H \text { Micro } \\
\text { ammed death-ligan } \\
\text { ed positive score. }\end{array}$ & $\begin{array}{l}\text { osatellite instabilit } \\
\text { ad } 1, I H C\end{array}$ & ty-high, \\
\hline
\end{tabular}

\section{Discussion}

The ICIs have been used in various treatment lines in clinical practice for patients with AGC. Herein, we analyzed 187 refractory advanced or recurrent GC patients with ICI, of whom 105 received ICI as a 3rd line and 82 received ICI as a 4th line treatment. The ORR for ICIs was $10.5 \%(11 / 105)$ in 3rd line and $8.5 \%(7 / 82)$ in 4 th line. The median PFS and OS to ICIs was not different between 3rd line and 4th line $(P=0.495$ and $P=0.208$, respectively). These finding suggested that ICI could be one of reasonable options at both 3rd line and 4th line therapies in refractory AGC patients.

Table 4. Univariate and multivariate analysis of overall survival after $\mathrm{ICls}$

\begin{tabular}{|c|c|c|c|c|c|c|}
\hline \multirow[t]{2}{*}{ Variables } & \multirow[t]{2}{*}{ No. } & \multirow{2}{*}{$\begin{array}{l}\text { Median OS (months, 95\% } \\
\text { CI) }\end{array}$} & \multicolumn{2}{|l|}{ Univariate Analysis } & \multicolumn{2}{|c|}{ Multivariate Analysis } \\
\hline & & & HR $(95 \%$ CI $)$ & $P$-value & HR $(95 \%$ CI $)$ & $P$-value \\
\hline Median age, years & & & & 0.018 & & 0.030 \\
\hline$<65$ & 140 & $2.7(2.2-3.3)$ & 1 & & 1 & \\
\hline$\geq 65$ & 47 & $2.6(0.0-5.5)$ & $1.61(1.08-2.38)$ & & $0.65(0.44-0.96)$ & \\
\hline Sex & & & & 0.265 & & \\
\hline Male & 109 & $3.1(2.4-3.8)$ & 1 & & & \\
\hline Female & 78 & $3.1(1.8-4.2)$ & $0.82(0.60-1.15)$ & & & \\
\hline Immune checkpoint inhibitors & & & & 0.209 & & \\
\hline $3^{\text {rd }}$ line & 105 & $4.4(1.6-7.2)$ & 1 & & & \\
\hline $4^{\text {th }}$ line & 82 & $2.8(2.2-3.4)$ & $1.23(0.89-1.70)$ & & & \\
\hline No. of metastatic sites & & & & 0.012 & & 0.019 \\
\hline$<2$ & 18 & $8.5(4.4-12.5)$ & 1 & & 1 & \\
\hline$\geq 2$ & 169 & $2.8(2.4-3.3)$ & $2.14(1.18-3.88)$ & & $2.05(1.13-3.71)$ & \\
\hline Microsatellite instability* & & & & 0.962 & & \\
\hline MSS & 148 & $7.4(4.8-10.1)$ & 1 & & & \\
\hline MSI-H & 2 & $4.8(2.1-7.4)$ & $1.04(0.26-4.20)$ & & & \\
\hline Not evaluable & 37 & & & & & \\
\hline EBV in situ & & & & 0.465 & & \\
\hline No & 118 & $3.3(2.6-4.1)$ & 1 & & & \\
\hline Yes & 9 & $6.2(0.0-16.1)$ & $0.75(0.35-1.63)$ & & & \\
\hline Not evaluable & 60 & & & & & \\
\hline $\begin{array}{l}\text { PD-L1 22C3 IHC } \\
\text { CPS } \geq 1\end{array}$ & & & & 0.931 & & \\
\hline No & 37 & $3.9(2.3-5.6)$ & 1 & & & \\
\hline Yes & 43 & $3.3(0.9-5.8)$ & $1.02(0.61-1.73)$ & & & \\
\hline Not evaluable & 107 & & & & & \\
\hline $\begin{array}{l}\text { PD-L1 22C3 IHC } \\
\text { CPS } \geq 10\end{array}$ & & & & 0.917 & & \\
\hline No & 66 & $3.1(2.5-3.8)$ & 1 & & & \\
\hline Yes & 14 & $3.0(0.8-5.3)$ & $1.04(0.53-2.01)$ & & & \\
\hline Not evaluable & 107 & & & & & \\
\hline
\end{tabular}


Previous studies have shown the efficacy and safety of ICIs as 2nd-, 3rd- or later line treatment in refractory GC patients, consistent with the present studies. For example, in the ATTRACTION-2 study, the median OS and PFS were 5.3 months and 1.6 months, respectively, and the ORR to nivolumab was $11 \%$ [6]. In the KEYNOTE-059 study, the median OS and PFS were 5.6 months and 2.0 months, respectively, and the ORR to pembrolizumab was $12 \%$ [7]. Systematic review and meta-analysis of ICIs reported that anti PD-1 inhibitor improved the long-term clinical benefit. However, ICIs were used as various treatment lines in previous studies [5]. Previous clinical trials showed subgroup analysis for the optimal timing of the application of ICIs. In the ATTRACTION-2 study, subgroup analysis for OS showed that patients with nivolumab as 4th-line treatment had better survival than patients with nivolumab in the 2 nd or 3rd line (Hazard ratio 0.44 versus 0.82 and 0.89 , respectively) [6], and the ORR of pembrolizumab was better in the setting of 3rd line than in 4 th or higher (ORR $16.4 \%$ versus $6.4 \%$ ) in the KEYNOTE-059 study [7]. The timing of the application of ICIs was different between two clinical trials. Based on our real world data, ICIs might be a reasonable option in both the 3rd line and 4th line for refractory GC patients.

Previous research has reported that ICIs were more effective in patients with PD-L1 positivity, MSI-H, or EBV positivity [5, 8, 9]. In the present study, 80 patients were assessed for expression of PD-L1 using CPS. There was no difference in PFS to ICIs according to the status of PD-L1 expression and between patients with EBV-positive and -negative tumors $(P=0.509)$. This discrepancy might be caused by heterogeneous patients' characteristics and small sample size.

In the present study, although ICIs were used as 3rd- or 4th-line therapy, 18 of 187 patients (9.6\%) achieved long-term survival of greater than 1 year. Furthermore, 7 of 187 patients (3.7\%) received ICIs for more than 1 year, and 3 continued to do so to present. This finding is concordant with previous studies reporting a long-term clinical benefit of ICI $[6,10,11]$. Considering that patients received ICIs as 3rd or 4th line therapy, these findings were very interested. Further, we must conducted the prospective biomarker research to select AGC patients who achieve the long term survival.

This analysis has limitations. First, it was a retrospective nature with a clinically heterogeneous population that is subject to potential biases. Second, the study included a relatively small number of patients, making it difficult to draw definite conclusions. Third, only Asian patients with GC were analyzed in the study, limiting generalizability because of differences in molecular profiles and clinical features between Western and Eastern patients with GC. Therefore, our findings must be interpreted with caution.

In conclusion, ICIs showed similar clinical outcomes between 3rd-line and 4th-line settings. ICIs might be a reasonable approach for refractory AGC patients in such settings.

\section{Abbreviations}

ICI: immune checkpoint inhibitors; GC: gastric cancer; OS: overall survival; PFS: progression-free survival; ORR: objective response rate; DCR: disease control rate; NCCN: National Comprehensive Cancer Network; ESMO: European Society for Medical Oncology; PD-1: programmed death-1; PD-L1: programmed death-ligand 1; MSI: microsatellite instability; EBV: Epstein-Barr virus.

\section{Author Contributions}

Conception and design: Hongsik Kim and Seung Tae Kim, Provision of study materials or patients: Hongsik Kim, Hana Kim, Minsang Lee, Minsuk Kwon, Jung Yong Hong, Jeeyun Lee, Ho Yeong Lim, Won Ki Kang and Seung Tae Kim, Collection and assembly of data: Hongsik Kim, Data analysis and interpretation: Hongsik Kim and Seung Tae Kim, Manuscript writing: All authors, Final approval of manuscript: All authors.

\section{Competing Interests}

The authors have declared that no competing interest exists.

\section{References}

1. Bray F, Ferlay J, Soerjomataram I, et al. Global cancer statistics 2018: GLOBOCAN estimates of incidence and mortality worldwide for 36 cancers in 185 countries. CA Cancer J Clin. 2018; 68: 394-424.

2. Hong S, Won YJ, Park YR, et al. Cancer Statistics in Korea: Incidence, Mortality, Survival, and Prevalence in 2017. Cancer Res Treat. 2020; 52: 335-350.

3. Smyth EC, Verheij M, Allum W, et al. Gastric cancer: ESMO Clinical Practice Guidelines for diagnosis, treatment and follow-up. Ann Oncol. 2016; 27 (Suppl 5): v38-v49.

4. [Internet] National Comprehensive Cancer Network. Gastric Cancer (Version 4. 2020). https://www.nccn.org/professionals/physician_gls/pdf/gastric .pdf.

5. Chen C, Zhang F, Zhou N, et al. Efficacy and safety of immune checkpoint inhibitors in advanced gastric or gastroesophageal junction cancer: a systematic review and meta-analysis. Oncoimmunology. 2019; 8: e1581547.

6. Kang YK, Boku N, Satoh T, et al. Nivolumab in patients with advanced gastric or gastro-oesophageal junction cancer refractory to, or intolerant of, at least two previous chemotherapy regimens (ONO-4538-12, ATTRACTION-2): a randomised, double-blind, placebo-controlled, phase 3 trial. Lancet. 2017; 390: 2461-2471.

7. Fuchs CS, Doi T, Jang RW, et al. Safety and Efficacy of Pembrolizumab Monotherapy in Patients With Previously Treated Advanced Gastric and Gastroesophageal Junction Cancer: Phase 2 Clinical KEYNOTE-059 Trial. JAMA Oncol. 2018; 4: e180013.

8. Kono K, Nakajima S, Mimura K. Current status of immune checkpoint inhibitors for gastric cancer. Gastric Cancer. 2020; 23: 565-578.

9. Kim ST, Cristescu R, Bass AJ, et al. Comprehensive molecular characterization of clinical responses to PD-1 inhibition in metastatic gastric cancer. Nat Med. 2018; 24: 1449-1458. 
10. Bang YJ, Ruiz EY, Van Cutsem E, et al. Phase III, randomised trial of avelumab versus physician's choice of chemotherapy as third-line treatment of patients with advanced gastric or gastro-oesophageal junction cancer: primary analysis of JAVELIN Gastric 300. Ann Oncol. 2018; 29: 2052-2060.

11. Shitara K, Özgüroğlu M, Bang YJ, et al. Pembrolizumab versus paclitaxel for previously treated, advanced gastric or gastro-oesophageal junction cancer (KEYNOTE-061): a randomised, open-label, controlled, phase 3 trial. Lancet. 2018; 392: 123-133. 